\title{
$616-85$ para el control del espesor del lecho de clínker en los enfriadores
} radioactive rays control clinker depth

D. H. GIESKIENG

(«Rock Products», febrero 1960, pag. 150.)

La gran energía de los rayos gamma emitidos por las sustancias radiactivas se ha aprovechado para desarrollar una misión importante en la industria del cemento. La medida de rayos gamma para controlar el espesor de una capa de material señala un gran avance en la automatización de los enfriadores de clínker del tipo de parrilla. Su misión es doble: preservar al enfriador de bruscas sobrecargas y mantener el proceso en el espesor de lecho máximo tolerable para un buen funcionamiento.

Con ello se imponen ventajas tanto sobre el clínker como sobre el horno, al estabilizarse la marcha y disminuir el costo de conservación. La temperatura de las placas se reduce y homogeneiza, se proporciona un "lecho muerto" que amortigua la caída del clínker sobre estas placas y la velocidad del enfriador se mantiene en un mínimo práctico. Además, el hornero conservará bien la marcha y podrá estabilizar prácticamente todas las perturbaciones del horno.

Aun cuando la mayor parte de los enfriadores de parrilla que se construyen se montan en fábricas de clínker de cemento, los principios que aquí se expliquen pueden tener interés en toda la industria de productos pétreos, tanto en lo que se refiere a las caracteristicas del enfriador como a los medios empleados en su control. De hecho, muchos de estos factores pueden aplicarse a otros tipos de enfriador.

La necesidad del control deriva de las oscilaciones en la producción del horno. El homo descarga clínker al extremo de alimentación de la parrilla enfriadora y se sopla aire a través de las placas y el lecho de material por medio de un ventilador, enfriando el clínker a medida que se transporta a lo largo de la máquina por efecto de un movimiento de sacudida. En la figura 1 , se representa un sistema clásico de enfriador.

Como las oscilaciones en la cantidad o características del material producido por el horno tienen influencia sobre este efecto transportador, las máquinas se suministran siempre con mecanismos que permiten una velocidad variable en el transporte. Unas instalaciones, por sus peculiaridades en la formación del clínker y marcha del horno, requieren más regulación que otras. Los primeros ensayos de automatismo en la velocidad de la parrilla (presión del aire en las cámaras inferiores, su temperatura, su velocidad de paso, etc.) no dieron resultados satisfactorios. Sin embargo, parece ser que la necesidad se completa con esta nueva aportación: el principio de medida del nivel por medio de rayos gamma para juzgar la profundidad o espesor de material en el enfriador.

La estabilidad de la temperatura tiene un punto de apoyo importante en el espesor del lecho. La compatibilidad del sistema horno-enfriador se afecta directamente por la estabilidad de la temperatura del aire secundario. Además, la economía de combustible se relaciona según una ley creciente con esta temperatura. El enfriador, por lo tanto, debe operarse en aquellas condiciones que proporcionan una temperatura de aire secundario muy estable. 


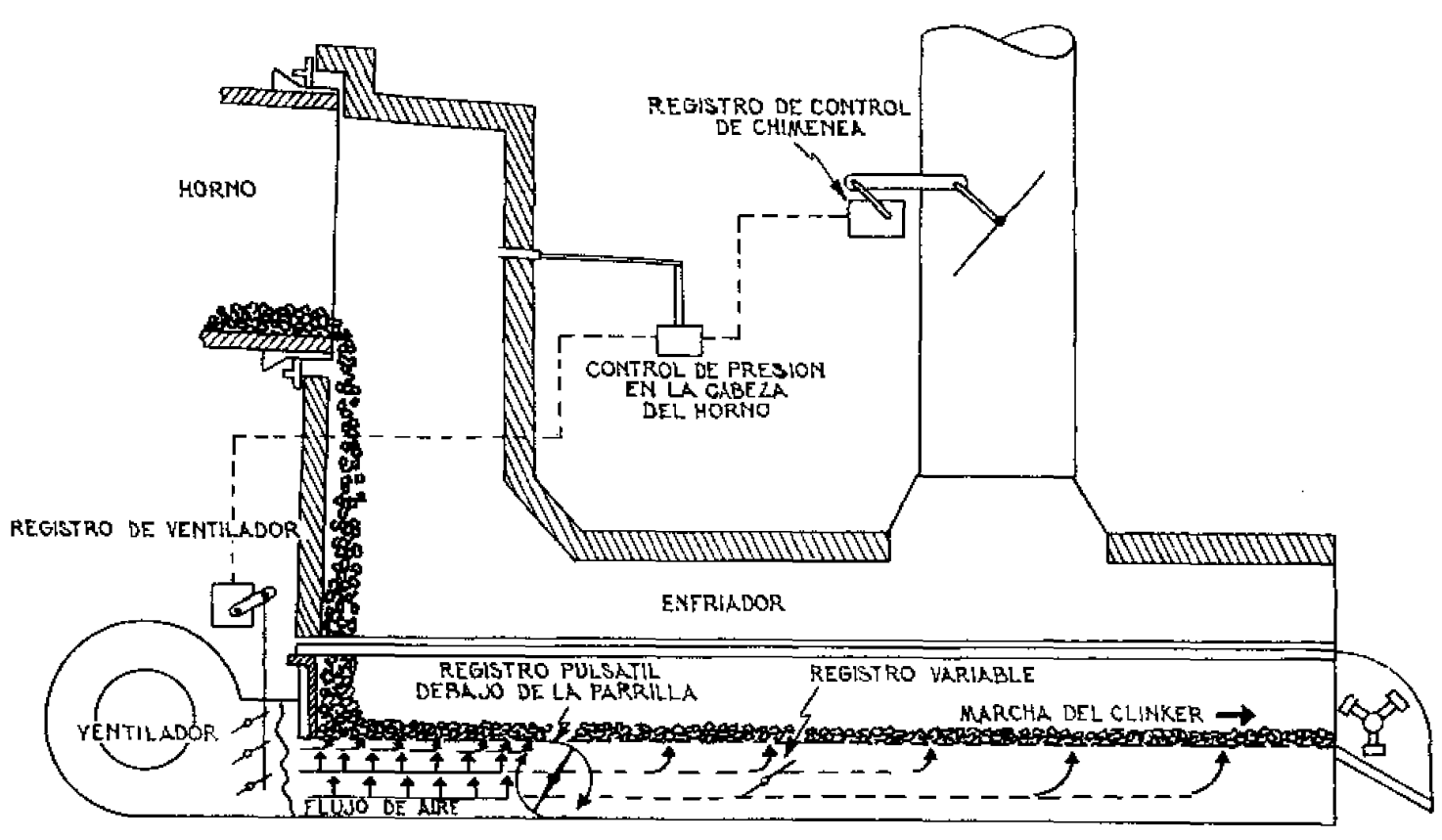

Fig. 1.-Parrilla entriadora del chinter con sus registros pulsátíl $y$ variable, idóneo para la splicacion del control nuelear de la profundtad del lecho.

Dependiendo de la naturaleza del clínker, cuyas caracteristicas se afectan por la composición del crudo y la temperatura, la acción conjunta de la parrilla y la ráfaga de aire refrigerante proporctonan una distribución uniforme del material hasta una cierta profundidad. Alcanzada esta profundidad, las fuerzas distributivas son insuflclentes, y el clínker, "amontonado" por la descarga del horno, puede mantener un lecho más espeso en el centro de las placas que en los costados (fig. 2).

Esta "1sła" tiene una mayor densidad en el centro y una permeabilidad relativamente más aita en los lados. Las áreas laterales se enfrian muy de prisa, produciendo una caída brusca en la temperatura del aire secundario, en tanto que la zona central permanece caliente y compacta.

La temperatura máxima estable de aire secundario se conseguirá con espesores de lecho ligeramente inferiores a aquellos que den lugar a la formación de esta "Isla".

En la flgura 3 se representa el efecto de la profundidad de lecho sobre la temperatura del alre secundario. Al aumentar el espesor, la temperatura del aire aumenta hasta un punto que se corresponde con la formación de la mencionada "isla". El cambio de pendiente en el punto A está influenciado por la temperatura y características del materíal; cuanto más caliente está el clínker, mayor será su tendencia a resistirse a la distribución y, en consecuencia, a formar "islas".

Los lechos estables de mayor espesor pueden conseguirse empleando un amortiguador pulsátil, con el que es posible operar en el punto B de la figura 3. El amortiguador es más eficaz cuando se sitúa en la cámara inferior del enfriador, donde se mantlene una presión de aire por debajo de las partillas de alimentación y a la que se superponen las ondas de presión en retroceso del amortiguador pulsátil. Esta pulsación brusca es muy efectiva en la distribución del material y en la creactón de una permeabilidad unlforme.

Por otra parte, esta estabilidad debe mantenerse a lo largo de todo el enfriador. La importancia de evitar islas y crear una permeabilidad uniforme a traves del lecho de clínker, tiene la contrapartida en la variación de profundidad a lo largo de dicho lecho. Lógicamente, cualquier automatización en la velocidad del enfriador seguiría paso a paso las variaciones de la producción del horno para igualar este efecto. Por ejemplo, si la producción aumenta bruscamente, como ocurre con la caida de un anillo, el control de velocidad del enfrtador seguirá inmediatamente esta variación, velocidad que debe colaborar en mantener el espesor y la permeabilidad uniformes a lo largo de todo el enfriador. 
La medida del nivel por medio de rayos gamma, cumplixá esta misión de control si se coloca el aparato en las proximidades del extremo de alimentación del enfriador. Sì se ajusta convenientemente, este control sigue las variaciones de producción a medida que tienen lugar.

Los cambios bruscos producidos por variación en la velocidad del horno son más acusados porque el efecto global es inmediato. Estos cambios pueden preverse por el hornero, quien puede modificar la velocidad del enfriador a la vez que cambia la velocidad del horno; mejor aún, puede establecerse un enclavamiento entre ambos controles, de modo que ambas velocidades se compensen automáticamente.

Cómo afecta el tamaño de los gránulos de clínker a la temperatura del aire secundario es, quizás, uno de los aspectos más contradictorios en el control de la profundidad del lecho. La discusión se reftere a las superflcies especiftcas de clínkeres grueso y fino, y a la norma de que el intercambio de calor es proporcional al área superficial.

Ya se sabe que el control de espesor, unido al control del flujo de aire secundario, resulta en una buena estabilización de las temperaturas de dicho aire.

El aire secundario es una mezcla del aire que procede de los primeros 4,5 a $6 \mathrm{~m}$ del enfriador. Esto significa que el clínker debe transmitir calor al aire de enfriamiento durante cuatro o clnco minutos, Los diagramas de las flguras 4 y 5 demuestran que debe tenerse en consideración la aptitud del clínker para sostener esta transmisión de calor.

Los cálculos del intercambio de calor pueden llegar a ser extremadamente complejos si se constdera el factor de "reserva de calor", tal como se muestra en la flgura 6 .

Establecido de un modo sencillo, significa que un grano de clínker de $2,5 \mathrm{~mm}$ de diámetro desprende su calor 10 veces más de prisa que uno de $25 \mathrm{~mm}$ y, en consecuencia,

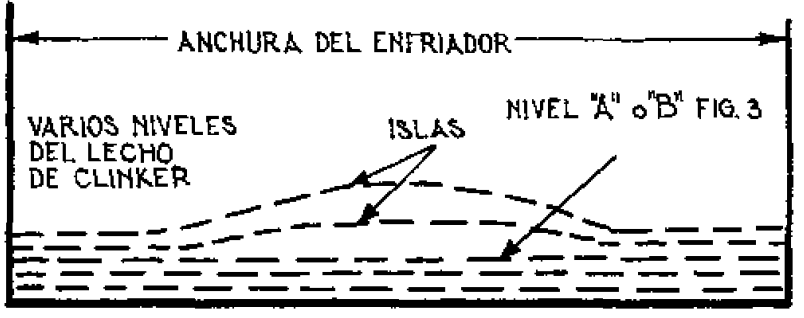

Fig. 2.-Una aislan de clinker caliente en el eentro del enfriador se resiste a la penetración del aire de refrigeración.
Fig. 3.-La profundidad del lecho de elinker puede ser mayor asando un registro pulsátil debajo de la parríla.

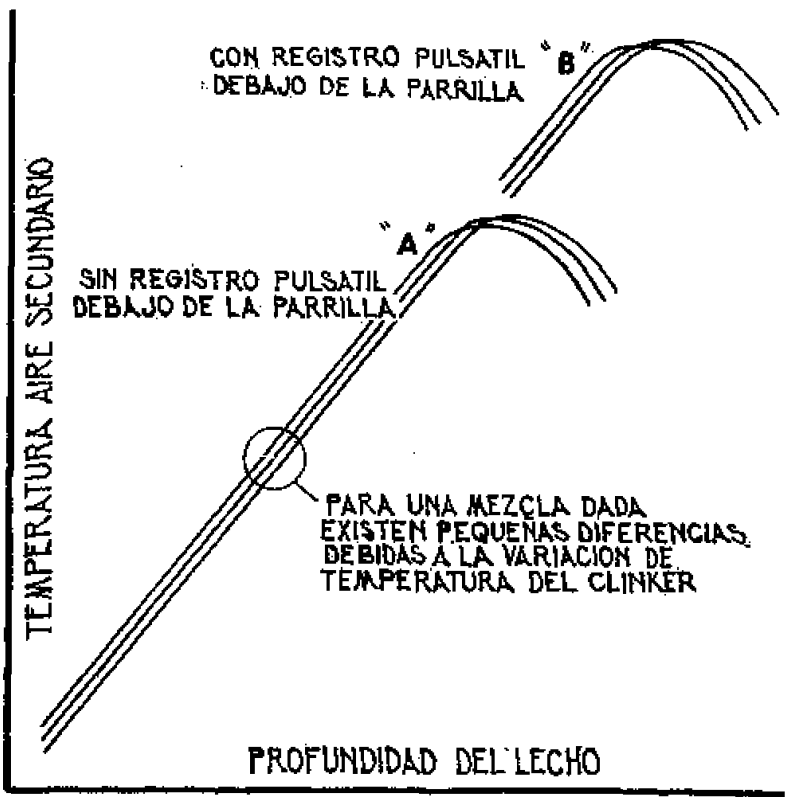




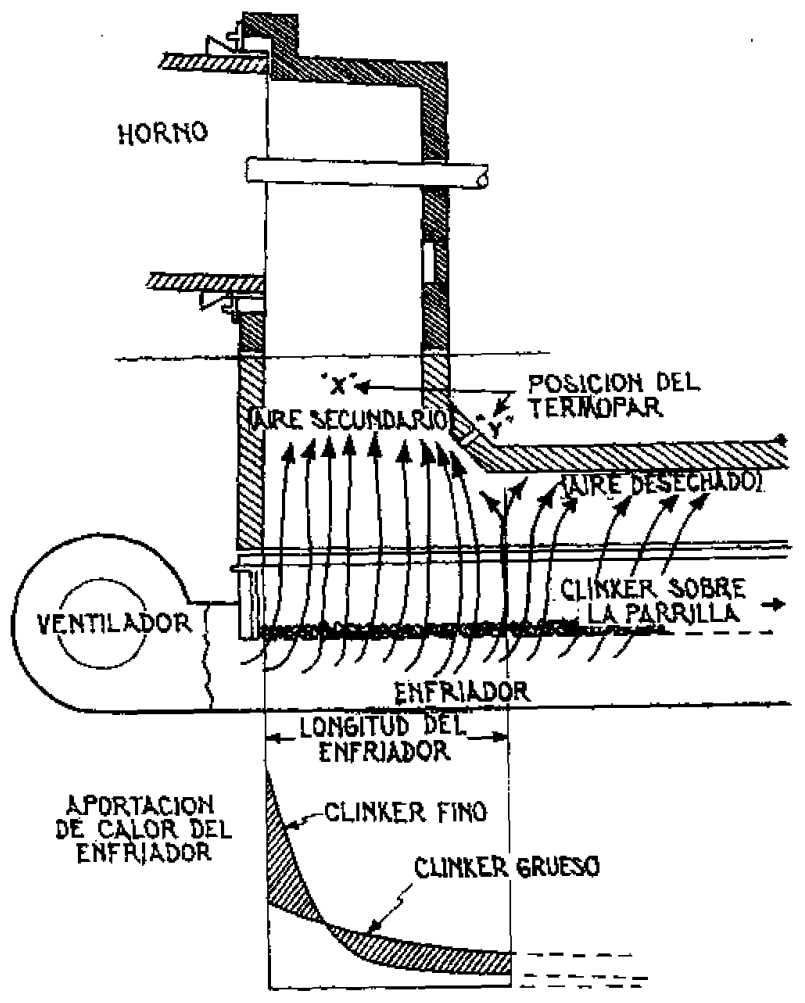

Figs. 4 y 5.-El clínker caliente aporta más calor al aire secmadario en ol primer tramo del enfriador $(1,5 \mathrm{~m}$ aproximadamente $)$

Fig. 6. - Para una profundidad dara $(X)$ dentro de las partículas Ftg.
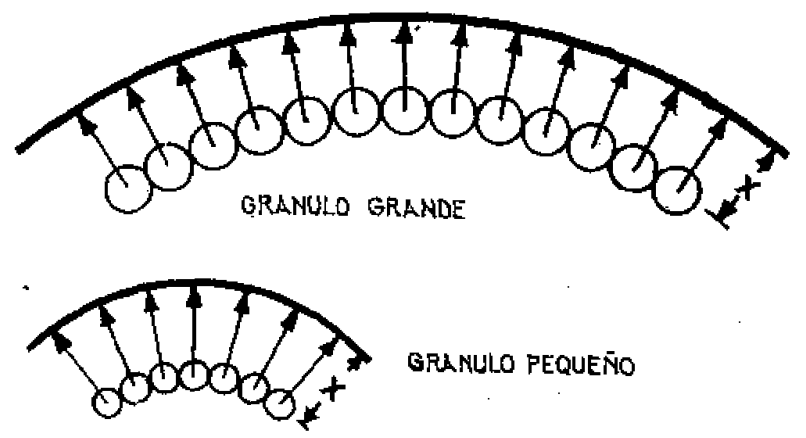

GRANLLO PEQUEÑO se enfriara mejor antes de abandonar la zona útil del aire secundario.

La figura 5 , compuesta con la flgura 4 , nos muestra la cesión de calor del clinker grueso y del clínker fino, y se observa siempre ina estabilidad excelente en lo referente a las variaciones de tamaño. si el enfriador se controla a una profundidad de lecho estable; esto requiere una descarga relativamente constante del horno, lo cual es normal. En el caso de un horno creando anillo, la velocidad de descarga varia. Puede observarse que, con una profundidad de lecho constante, la temperatura del aire secundario global crecerá al aumentar la descarga del horno, ya que con mayor velocidad de transporte se arrastrará clínker más caliente hacia el final de la zona que proporciona el aire secundario. Como este cambio en la temperatura del aire secundario tiene lugar en el sentido adecuado para mantener "satisfecha la carga", y viceversa, tenderá a estabilizar las condiciones en la zona de cocción durante el periodo de formactón del anillo.

La medida de la temperatura del aire secundario se realiza por termopares de aspiractón. En la flgura 5 se observa que la posición de un termopar de este tipo en la entrada del enfriador puede modiflcar sus indicaciones. Aunque para obtener una "posición media" verdadera deben considerarse el efecto de la raíz cuadrativa media $y$ otros, una posición práctica podia ser el punto medio del costado de la boca del enfriador (punto $x$, ffgura 4).

Se ha conseguldo una estabilit. zación notable de las temperaturas del aire secundario, medidas con termopares de aspiraclón, comblnando el control del espesor del lecho y el del ventilador.

Otra demostración de estos factores estabillzantes ha sido el registro del porcentaje de oxígeno en los gases de escape del horno. A constancia de otros factores (suministro de combustible y tiro del horno), los kilos de aire que entran en el horno serán inversamente proporclonales a la temperatura absoluta del aire secundario. Al cambiar la temperatura 
media del aire secundario de 650 a $595^{\circ} \mathrm{C}$ (un cambio de $55^{\circ}$ ), se aumentó el peso del aire en un $6 \%$. Esto resultó en un cambio de un $1 \%$ en el análisis de los gases de escape.

La figura 7 es un diagrama típico de análisis de oxígeno antes de instalar el control de profundidad del lecho, aunque con registro en el ventilador de la parrilla.

La flgura 8 es el mismo diagrama después de instalar dicho control: se observan variaciones inferiores a $0,2 \%$ de $\mathrm{O}_{2}$ durante largos períodos de tiempo. La variación de la temperatura del aire secundario total en estos períodos de tíempo, por su relación con el analizador de oxígeno, sería inferior a $11^{\circ} \mathrm{C}$, permitiendo pequeñas variaciones en la velocidad de cocción, etc.

Las temperaturas del aire gecundario aspirado durante este ensayo se hallaban en una banda de $42^{\circ} \mathrm{C}$; no obstante, el termopar se encontraba colocado en $\gamma$ (figura 4), y, por consiguiente, esto no conducía a promediar el efecto de la variación de tamaño del clínker (fig. 5).

Como antes, se han mantenido constantes otros efectos, tales como la velocidad de cocción y el tiro del horno; de lo contrario, el efecto de temperatura sobre el análisis de oxígeno estaría enmascarado.

El control de presión en la cabeza del horno se lleva a cabo con el ventilador de la parrilla y con el registro del aire que se expulsa por la chimenea del enfriador.

El enfriador, del cual se representaron las gráficas (figs. 7 y 8), tenía un tiro natural por chimenea con registro fijo, y la presión en la cabeza del horno se automatizaba con el registro del ventilador de la parrilla.

Esta automatización tiene un efecto estabilizador mayor que con la automatización en el registro de la chimenea. Esto puede ser debido a una acción directa sobre la variable controlada o la tendencia a suministrar más presión debajo de la parrilla, siempre que una dis-

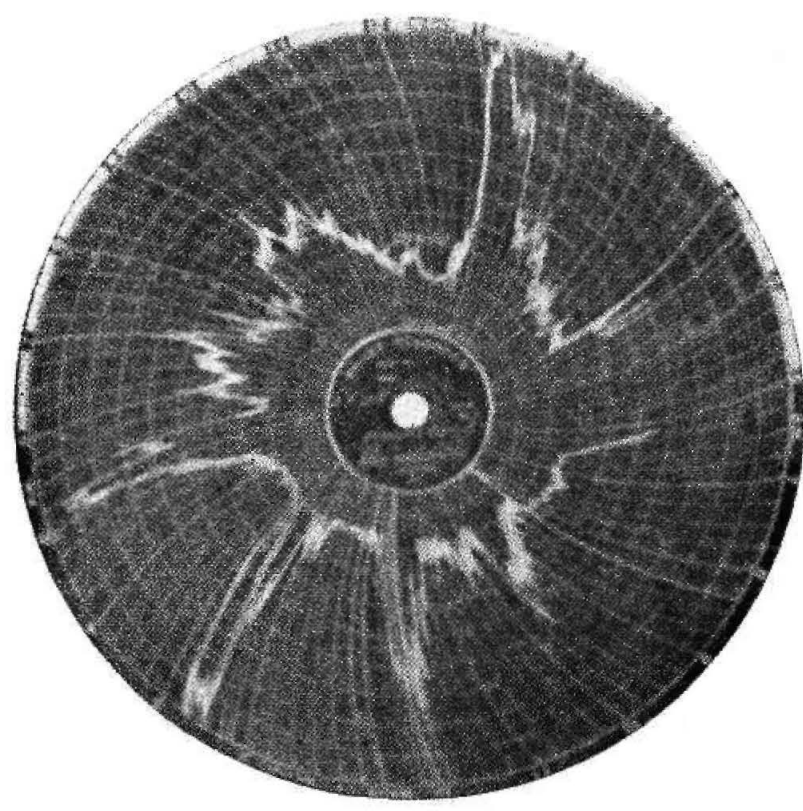

Fig. 7.-Curva típica del analizador de oxígeno en un enfriador accionado a mano.

Fig. 8-El control automático del enfriador de clinker elimina las desviaciones en el análisis de oxígeno.

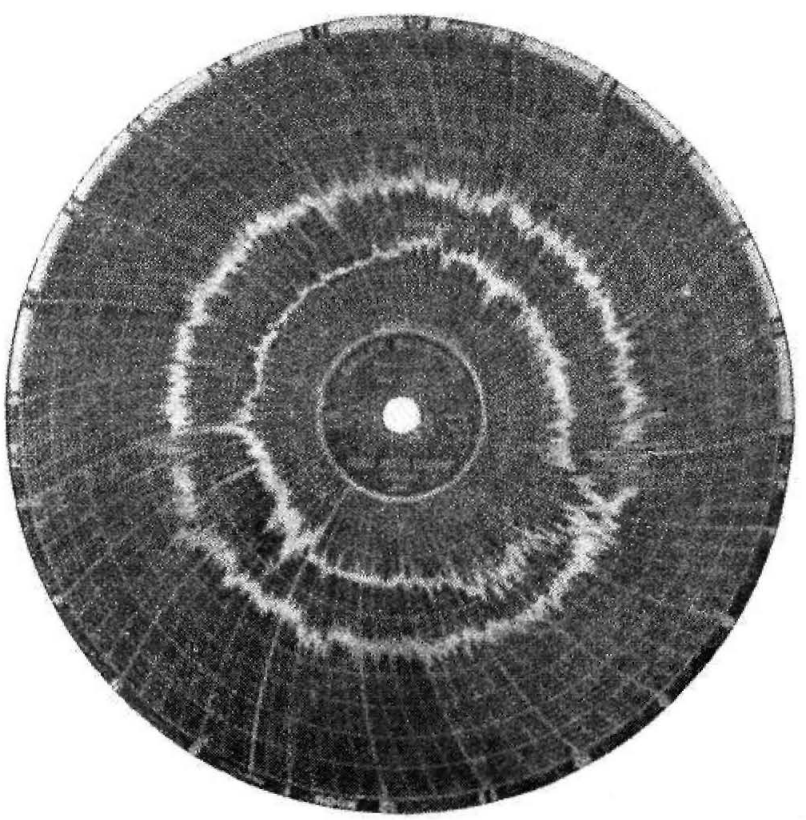


VENTANA

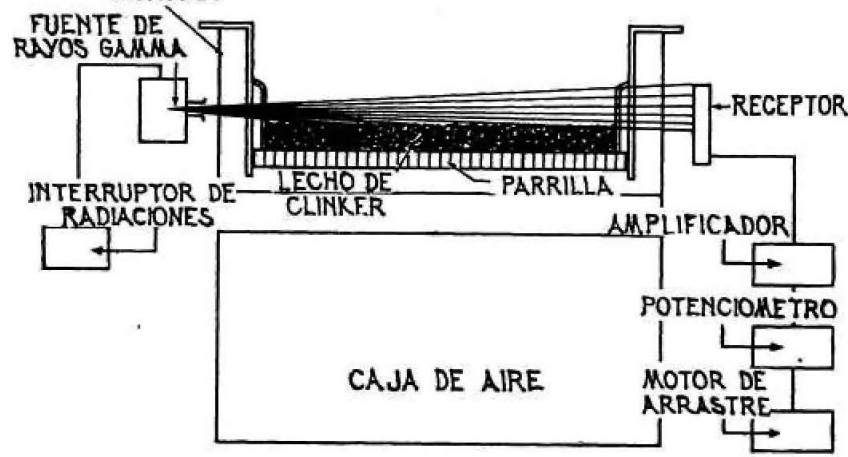

Fig. 9.-Una sección transversal del enfriador muestra los componentes principales del nuevo control, fuente de radiación y receptor Geiger-Mueller. minución de permeabilidad del lecho lo requiera.

Todas las consideraciones indican la necesidad de una variación simultánea del registro del ventilador y del registro de la chimenea para controlar la presión en la cabeza del horno.

El enlace entre el enfriador y los registros de tiro sería simultáneo $\mathrm{y}$ opuesto, $\mathrm{y}$ daría lugar al movimiento proporcional ajustable entre los dos, teniendo uno de los registros una acción de ajuste mayor que el otro. Los niveles relativos o inclinaciones de las válvulas serian también ajustables de modo que pueda obtenerse la combinación óptima para una instalación dada.

Por ejemplo, mientras el registro del ventilador de la parrilla varia de un 100 a un $60 \%$ de apertura, el registro de chimenea puede moverse, simultáneamente, de 50 a $70 \%$ de apertura. En otras instalaciones, una combinación ideal puede ser variar de 70 a $100 \%$ la apertura del registro de chimenea y de 100 a $60 \%$ la del registro del ventilador de parrilla.

El control de ambos registros por medio de un instrumento común reduce las oscilaciones y proporciona una transición suave con cambios en la permeabilidad del lecho del clínker.

Esto significa que, a medida que aumenta el tamaño del clínker, más aire pasará a través de la última zona del enfriador (más que cuando el registro de chimenea está fijo y sólo se varia el registro del ventilador). Al existir más depresión sobre la parrilla al final del lecho de clínker, se colaborará en el enfriamiento de este material, que, naturalmente, enfría más despacio.

La medida del nivel por medio de los rayos gamma como una manera de saber la altura del lecho es conocida desde hace unos diez años, pero su primera aplicación a un enfriador de parrilla se hizo en octubre de 1958. Las ventajas derivan del hecho de que puede colocarse fuera del cuerpo del enfriador y próximo al extremo de caída del horno para darnos una primera referencia. 
Fig. 11. - Receptor $y$ amplificador del control nuclear de proe in e instala pegado a la par del enfriador.

Desde la introducción del medidor gamma se han hecho notables mejoras en los tubos GeigerMueller, y el aparato está prácticamente libre de gastos. En la figura 9 se esquematiza el principio de funcionamiento de! contador. Un manantial, adecuadamente protegido, envía un haz de rayos gamma a través de las paredes laterales del enfriador, placas y revestimientos. Con excepción del efecto de interferencia del clínker, estos rayos llegan debilitados al lado opuesto, aunque de un modo uniforme. A medida que varía el nivel de clínker, se "sombreará" más o menos el fondo de los tubos Geiger - Mueller. Esto aumenta o disminuye la salida de estos tubos, la cual se amplifica y aparece en un potenciómetroregistro standard.

El potenciómetro de control ajusta automáticamente la velocidad del enfriador para mantener constante el nivel del lecho; si éste aumenta, la velocidad del enfriador crece paulatinamente $y$ viceversa.

En la figura 10 se muestra un manantial de rayos gamma montado próximo al enfriador. La caja que encierra la fuente de rayos está preparada de modo que, si se para el motor del enfriador, se enciende una luz y funciona un sistema de alarma hasta que dicha caja vuelve a su posición de "cerrada". Si se observa la entrada del enfriador, la caja se mantiene cerrada (tal como exigen las Normas de la Comisión de Energía Atómica).
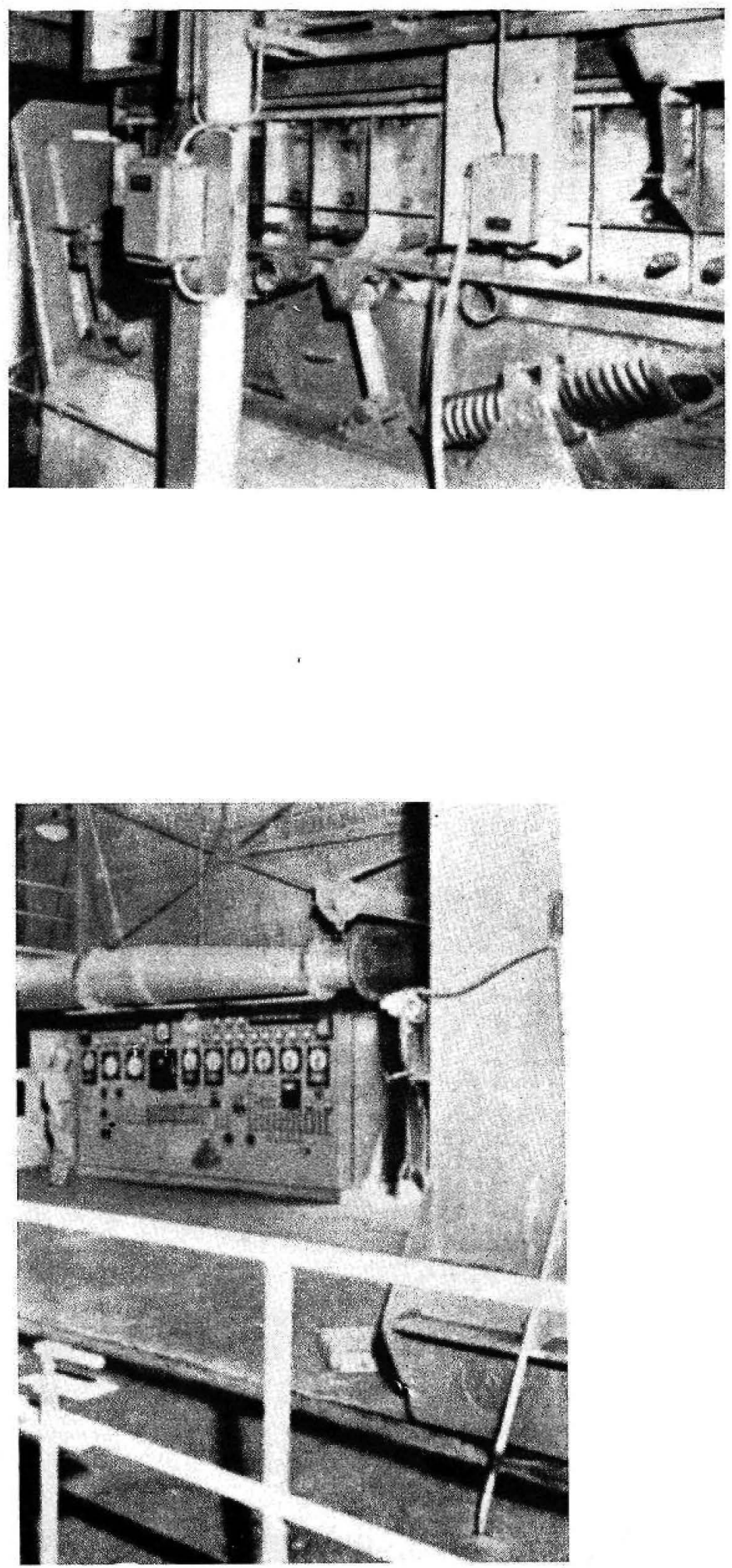

Fig. 12.-Plataforma de hornero en una moderna fábrica de cemento; en el panel se controla la apertura $y$ cierre del transmisor radiactivo para el control nuclear de la profundidad del lecho. Aqui se controla tanto la velocidad como la profundidad del lecho en el enfriador de 
Este es un instrumento seguro. Los campos de radiación alrededor de la caja y en el lado de recepción de rayos son pequeños y se encuentran inclufdos perfectamente dentro de los límltes aceptados por dicha Comislón. El campo más intenso dentro del enfriador está protegido, suponiendo que nadie entre mientras se encuentra en funcionamiento dicho enfriador, $y$ el sistema de alarma cerrará la fuente si el enfriador se para.

En la flgura 11 se observará una caja que contlene el tubo Geiger-Mueller, montada junto al enfrlador. El amplificador está adosado en la columna de la izquierda. La figura 12 nos muestra el manejo de la operación a distancia y, por tanto, el hornero puede cerrar la fuente de radiaclones desde la plataforma del horno.

Tanto el enfriador como el clínker que de él sale, no toman radiactividad como consecuencia del empleo de rayos ganma.

Para controlar los enfriadores se usaba antes la medida de la presión debafo de la rejilla. De un modo general, la mayor parte de las dificultades con este sistema parece ser que se debian a su incapacidad para reconocer las variaciones en el tamaño del clínker y las interrupciones en la marcha de la permeabllidad, cuando se operaba en las proximidades de los puntos A y B Te la flgura 3.

(A) Bajo ciertas condiciones (corrientemente temperaturas de coccion excesivas), es posible hacer crecer el tamaño del gránulo de clínker, eliminando la presencia de flnos. Esto resulta en una permeabilldad de lecho extremadamente alta. Controlando sólo la presión bajo las placas, se sobrecargan los enfriadores en ocasiones de este tipo.

(B) La flgura 5 indica la conveniencia de mantener constante el volumen sobre las placas para hacer posible una aportación de calor media. Si se controla una permeabilidad constante, por la presión bajo la rejilla, se perturba esta conveniencia; un lecho de clinker fino será más delgado y uno de clinker grueso más espeso, tendiendo a agravar las perturbaciones del horno. Muchas fábricas que controlan la presión bajo la rejilla han comprobado esto, consiguiendo excelentes gráficos en la presión, pero, simultảneamente, han obtentdo gráficos de temperatura de aire secundario deficientes.

(C) Como ya se ha señalado, interesa llevar el enfriador en las proximidades de la "profundidad máxima de lecho estable". Volviendo a las figuras 2 y 3 , sabemos que la permeabilidad puede cambiar bruscamente poco antes de llegar a los puntos A o B y su repercusión en la presión bajo la rejilla no estaría de acuerdo con los objetivos de un control estable. Las instalaciones que van bien por el control de dicha presión, marchan quizás por debajo de la profundidad máxima de Iecho estable.

En casi todos los casos de perturbaciones en el horno, aun con operarios experimentados, es necesaric suprimir el control automático y volver al control a mano.

A pesar de estas defietencias, los más avezados operarios en ei control de la presión bajo la parrilla han conseguido un trabajo rutinario y una conservación reducida, en comparación con el trabajo manual.

Al emplear el contador gamma para controlar la profundidad de lecho, se marcha bien y se establlizan prácticamente todas las perturbaciones del horno. La única excepción es cuando el horno marcha frío y no clinkeriza; en este caso, el material crudo se criba en la rejilla y no permite alcanzar el punto correcto. Entonces, conviene volver al control manual hasta que se consiga una clinkerización perfecta.

Como cuando el horno marcha bajo de temperatura la velocidad del enfriador es baja, debe instalarse una luz de advertencia para llamar la atención del hornero, el cual hará todo lo posible para evitar estas situaciones.

Es muy conveniente, st se quiere conseguir el Ináximo rendimiento en el control de la capa de clínker, mantener el automatismo en el registro del ventilador de la parrilla y en el amortiguador pulsátil. Este último es, de todos modos, el más importante y una salvaguarda constante contra las sobrecargas. Mantiene la marcha en las proximidades de la profundidad de lecho máxima estable, reduciendo y equilibrando las temperaturas en la parrilla. Proporciona un lecho perfecto para almohadillar la caida de clinker en las placas y conservar la velocidad del enfriador lo más baja posłble. Estas condiclones de operación ideales se han conseguido en la práctica $\mathrm{y}$ han derivado en costes de operaciones notablemente reducidos. 